\title{
Enter the dragon
}

In Proc. 2020 IEEE Symposium on VLSI Technology (in the press); https://go.nature.com/38FQLhw

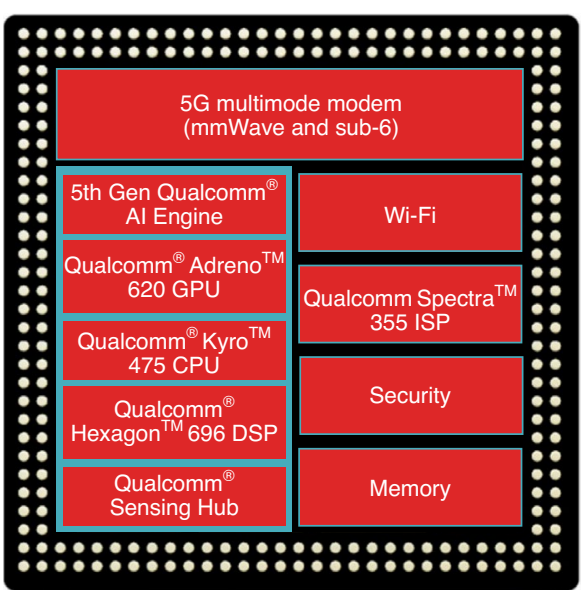

Credit: IEEE

Systems on a chip (SoC) are integrated circuits that contain numerous components, such as processors, memory, mixed signal and communications systems, on a single substrate or 'chip'. Their extensive use in mobile communications devices, including smartphones and tablets, has been one of the key drivers of complementary metaloxide-semiconductor (CMOS) technology scaling. The roll-out of $5 \mathrm{G}$ communications and artificial intelligence (AI) technologies, and the ever-increasing demands from consumers, means that SoCs require further improvements in performance, efficiency and functionality. Jie Deng and colleagues at Samsung Electronics and Qualcomm Technologies have now reported their latest mobile SoC platform that is integrated with $5 \mathrm{G}$ and $\mathrm{AI}$ technologies.

The researchers manufactured their SoC - termed Snapdragon 735 - using extreme ultraviolet lithography (EUV)-based $7 \mathrm{~nm}$ FinFET technology. The approach provides a $5 \%$ chip area reduction over previous non-EUV $7 \mathrm{~nm}$ technology, and a $35 \%$ area reduction over $10 \mathrm{~nm}$ technology. The SoC supports millimetre-wave and sub- $6 \mathrm{GHz}$ bands of 5G. It contains custom graphics, signal, and image processors, an AI engine, as well as a multi-core central processing unit (CPU) and memory. Though details in the paper are limited, process and design optimization enabled lower voltage CPU operation, contributing to an overall $20 \%$ increase in performance and $35 \%$ decrease in power consumption compared to their nearest predecessor SoC, the Snapdragon 730.

\section{Stuart Thomas}

Published online: 21 July 2020

https://doi.org/10.1038/s41928-020-0456-2 